\title{
BIOMASSA DA PARTE AÉREA DA VEGETAÇÃO DA FLORESTA TROPICAL UMIDA DE TERRA-FIRME DA AMAZÔNIA BRASILEIRA.
}

\author{
Niro HIGUCHI ${ }^{1}$, Joaquim dos SANTOS ${ }^{1}$, Ralfh João RIBEIRO', Luciano \\ MINETTE', Yvan BIOT ${ }^{2}$
}

\begin{abstract}
RESUMO - Usando um banco de dados com 315 árvores, com DAP $\geq 5 \mathrm{~cm}$, foram testados quatro modelos estatísticos - linear, não linear e dois logaritmicos - para estimar a biomassa de árvores em pé. Os dados foram coletados, de forma destrutiva, na região de Manaus, Estado do Amazonas, em um sítio coberto por floresta de terra-firme sobre platôs de latossolo amarelo. Em diferentes simulações com diferentes intensidades de amostragem, os quatro modelos estimam precisamente a biomassa, sendo que o afastamento entre a média observada e a estimada, em nenhuma ocasião ultrapassou $5 \%$. As equações para estimar a biomassa de árvores individuais em uma parcela fixa, distintamente para árvores com $5 \leq \mathrm{DAP}<20 \mathrm{~cm}$ e com DAP $\geq 20 \mathrm{~cm}$, são mais consistentes do que o uso de uma única equação para estimar, genericamente, todas as árvores com DAP $\geq 5 \mathrm{~cm}$. O modelo logarítmico com apenas uma variável independente, 0 DAP, apresenta resultados tão consistentes e precisos quanto os modelos que se utilizam também da variável altura total da árvore. Além do modelo estatístico para estimar o peso da massa fresca total de uma árvore, outras informações são apresentadas, estratificadas nos diferentes compartimentos (tronco, galho grosso, galho fino, folhas e, eventualmente, flores e frutos) de uma árvore, como: concentração de água para estimar o peso da massa seca, concentração carbono e a contribuição do peso de cada compartimento no peso total.
\end{abstract}

Palavras-chaves: Carbono, manejo florestal, modelo estatistico, alometria.

\section{Aboveground Biomass of the Brazilian Amazon Rainforest.}

ABSTRACT - A data set with 315 trees with diameter at breast height (dbh) greater than $5 \mathrm{~cm}$ was used to test four statistical models - linear, non-linear and two logarithmics - to estimate aboveground biomass of standing trees. The data were collected destructively near Manaus, Central Amazonia, on a site covered by a typical dense "terra-firme" moist forest on plateaus dominated by yellow oxisols. The difference between observed and estimated biomass was always below $5 \%$. The logarithmic model using a single independent variable (dbh) produced results as consistent and precise as those with two variables (dbh and total height). Besides statistical models to estimate aboveground biomass, the following information are also presented in this paper: the contribution of each tree component (stem, branch, twigs, leaves and flowers or fruits) to the total weight of a standing tree, water content to estimate the dry weight and carbon concentration of each tree component.

Key words: Carbon, forest management, statistical models, allometry.

\section{INTRODUÇÃO}

O objetivo deste trabalho é o desenvolvimento de modelos estatísticos para estimar a biomassa individual, de árvores em pé, de espécies da floresta densa de terrafirme, região de Manaus (AM), assim como a apresentação de informações necessárias para a conversão de massa fresca para massa seca e de biomassa para estoque de carbono. São testados quatro modelos, linear, não-linear e dois logaritmicos, tendo como variável dependente, o peso da massa fresca (não seca) e, como variáveis independentes, diâmetro à altura do peito (DAP) e altura total, de árvores

\footnotetext{
1 Instituto Nacional de Pesquisas da Amazônia - Cx. Postal, 478 - Manaus - Am.

2 U. K. Overseas Development Administration (ODA). Victoria Street, 94 - London. SW1E5JL - England.
} 
individuais. $\mathrm{O}$ principal atributo dos modelos testados é o tamanho da árvore e, por esta razão, têm que absorver a alta diversidade floristica e as diferentes associações botânicas, distribuições espaciais e densidades da madeira (intra e interespecíficas), da vegetação de terra-firme.

As estimativas de biomassa florestal são informações imprescindíveis nas questões ligadas, entre outras, às áreas de manejo florestal e de clima. No primeiro caso, a biomassa estả relacionada com os estoques de macro e micronutrientes da vegetação, que são obtidos pelo produto da massa pelas concentrações de cada mineral. No caso do clima, a biomassa é usada para estimar os estoques de Carbono, que, por sua vez, são utilizados para estimar a quantidade de $\mathrm{CO}_{2}$ que é liberada à atmosfera durante um processo de queimadas.

O manejo florestal está associado ao uso sustentável dos recursos florestais existentes, para atender às demandas da sociedade, por produtos madeireiros e não-madeireiros. Tratando-se de Amazônia, os cuidados têm que ser redobrados porque estes recursos estão em ecossistemas heterogêneos, complexos e frágeis. Os solos da Amazônia são antigos e, em sua maioria, pobres em nutrientes (especialmente para a agropecuária) e ácidos. A contrastante exuberância de sua cobertura florestal está associada às estratégias de conservação e de ciclagem de nutrientes dentro do próprio sistema. É importante conhecer a distribuição de nutrientes nos diferentes compartimentos (tronco, galho, casca, folha), para controlar a exportação dos mesmos pela colheita florestal e entrada via "inputs" atmosféricos e, com isto, minimizar os impactos ambientais da produção madeireira.

Para as questões climáticas, há grande interesse em quantificar a biomassa que é convertida, principalmente em dióxido de carbono, pelas diferentes formas de uso do solo amazônico (Fearnside et al., 1993; Foster Brown et al., 1995; Higuchi \& Carvalho Jr., 1994; Skole et al., 1994; Schroeder \& Winjum, 1995 ; Fearnside, 1996). Esta informação é necessária para uma correta avaliação da contribuição dos projetos de desenvolvimento da região, no processo de mudanças climáticas globais, no âmbito da Convenção do Clima, assinada pelo Governo Brasileiro durante a Conferência das Nações Unidas sobre Desenvolvimento e Meio Ambiente, Rio-92.

As estimativas de biomassa, atualmente disponiveis na literatura, dos diversos tipos florestais da Amazônia, vêm de estudos que se utilizam de métodos diretos e indiretos. O método direto consiste na derrubada e pesagem de todas as árvores que ocorrem em parcelas fixas, fornecendo estimativas, que segundo Brown et al. (1989), não são confiáveis porque baseiam-se em poucas parcelas, pequenas e tendenciosamente escolhidas. No método indireto, as estimativas têm sido produzidas a partir de dados de inventários florestais, que foram executados com a finalidade de planejar a exploração e o manejo 
florestal, sendo o volume da madeira, a principal variável. Neste método, a biomassa é estimada a partir do volume da madeira, usando-se a densidade média da madeira e um fator de correção para árvores com $\mathrm{DAP}<25 \mathrm{~cm}$.

Estes dois métodos ainda geram muita polêmica e controvérsias e produzem estimativas desencontradas, mesmo quando se usa o mesmo banco de dados (Fearnside et al., 1993; Brown et al., 1989; Higuchi et al., 1994; Foster Brown et al., 1995). A tabela 1 ilustra o que foi posto anteriormente. Esta tabela foi parcialmente reproduzida de Fearnside $e t$ al. (1993), considerando apenas a biomassa viva acima do nivel do solo. São produzidas estimativas diferentes, com o passar do tempo, pelos mesmos autores e para o mesmo banco de dados (montado nos anos 70). Além disso, Foster Brown et al. (1995) criticam estes bancos de dados, afirmando que as alturas das árvores foram obtidas sem aparelhos de mediçẫo e que, estes erros não amostrais não são mencionados.

O consenso existente entre os pesquisadores que trabalham com biomassa é de que é praticamente impossível determinar a biomassa de cada árvore, pelo método direto, ao executar um inventário florestal. Por esta razão, os recursos da análise de regressâo para o desenvolvimento de modelos estatísticos, para estimar a biomassa de árvores em pé, devem ser empregados para superar este problema. Salomão et al. (1996) citam apenas dois modelos estatísticos utilizados na Amazônia; um proposto por Sandra Brown e colaboradores e, outro, proposto por Christopher Uhl e colaboradores. $\mathrm{O}$ primeiro requer $\mathrm{o}$ conhecimento da densidade da madeira de cada indivíduo, que é praticamente impossível obte-la durante o inventário; e o segundo, é recomendado para florestas secundárias. Além destes, há o modelo de Overman et al. (1994), para a floresta amazônica colombiana, desenvolvido principalmente para árvores de pequenos diâmetros.

\section{Materiais e Métodos}

\section{(i) Coleta de Dados:}

Os dados foram coletados na Estação Experimental de Silvicultura Tropical (EEST) do INPA, aproximadamente $90 \mathrm{~km}$ ao norte de Manaus, em áreas derrubadas para experimentos com liberação de dióxido de carbono, usando-se queimadas tradicionalmente praticadas por

Tabela 1. Algumas estimativas de biomassa para a floresta densa da Amazônia brasileira*.

\begin{tabular}{cccc}
\hline Tipo de floresta & local & biomassa (t) & fonte \\
\hline Densa (RADAMBRASIL) & Amazônia & 268 & Brown \& Lugo (1992a) ) - cf. fonte \\
Densa (FAO) & Amazônia & 162 & Brown \& Lugo (1992a) - cf. fonte \\
Densa (RADAMBRASIL) & Amazônia & 289 & Brown \& Lugo (1992b) - ct. fonte \\
Densa (FAO) & Amazônia & 227 & Brown \& Lugo (1992b) - cf. fonte \\
Densa (presente) & Amazônia & 142,3 & Fearnside (1992a) - cf. fonte \\
Densa (presente) & Amazônia & 319,9 & Fearnside (unpub. 1993) - cf. fonte \\
\hline
\end{tabular}

(*) Fonte: parcialmente reproduzida de Fearnside et al. (1993) 
pequenos produtores da região, e em áreas especialmente designadas para esta pesquisa. Nos dois casos foram escolhidas áreas de platôs sobre latossolo amarelo. Estes dados constituem o banco de dados de biomassa do INPA.

No total, foram derrubadas e pesadas 315 árvores-amostras com DAP $\geq 5 \mathrm{~cm}$. O peso total de todos os indivíduos amostrados foi compartimentado em tronco e copa (incluindo galhos e folhas e, eventualmente, frutos). Além do peso da árvore, foram também medidos o DAP, altura total, altura comercial, altura da copa e diâmetro da copa. A distribuição de freqüência e a estatistica descritiva dos dados observados encontram-se nas tabelas 2a e 2b. Na tabela 2c observam-se as estatísticas descritivas para as variáveis DAP, altura total e peso total, quando os dados são divididos em algumas classes de diâmetro. Nesta tabela fica evidente que a variável peso total tem uma variabilidade natural bem maior que as outras duas variáveis, mesmo em mais classes de diâmetro.

Para obtenção das concentrações de água e nutrientes de cada compartimento das árvores, 38 indivíduos (dos 315 amostrados) foram coletados diferentemente, baseando-se no esquema apresentado por Higuchi \& Carvalho Jr. (1994) e Santos (1996). Foram retiradas amostras (discos) a 0\% (base), 25, 50, 75 e $100 \%$ (topo) do tronco e do galho grosso (diâmetro de base $10 \mathrm{~cm}$ ). Do tronco foi retirado também um disco à altura do DAP. Todos os discos retirados foram imediatamente pesados e enviados ao laboratório para secagem em estufas calibradas a $105^{\circ} \mathrm{C}$. O mesmo procedimento foi adotado para os galhos finos e folhas, mas que em vez de discos, foram retiradas, de várias partes da copa, amostras de 5 e $3 \mathrm{~kg}$, respectivamente. A estimativa da concentração de carbono na vegetação das espécies mais abundantes, no sítio estudado, foi feita tendo ainda as amostras coletadas por Higuchi \& Carvalho Jr. (1994).

O peso total de cada uma destas 38 árvores foi compartimentado em tronco, casca, galho grosso, galho fino (diâmetro $<10 \mathrm{~cm}$ ), folha e, eventualmente, flores e frutos. Além destas concentrações, a coleta compartimentada permite ainda a determinaçã̃o da contribuição de cada um dos compartimentos no peso total da árvore. A estatística descritiva destes dados e a contribuição de cada compartimento no peso total e a percentagem do Peso da massa fresca que é transformado em Peso da massa seca, visualizam-se nas tabelas $3 \mathrm{a}$ e $3 \mathrm{~b}$.

Um desdobramento da pesquisa de Nutrientes é o estudo de densidade da madeira $\left(\mathrm{g} / \mathrm{cm}^{3}\right)$, nos sentidos base-topo e casca-medula da árvore (utilizando-se das amostras coletadas a $0,25,50,75$ e $100 \%$ da altura comercial e do DAP). Resultados preliminares deste estudo encontram-se na tabela 4, de 12 árvores analisadas.

$\mathrm{O}$ banco de dados de biomassa do INPA vem sendo completado ao longo do tempo e já foi utilizado preliminarmente por Higuchi et al. (1994), Higuchi \& Carvalho Jr. (1994), Araújo (1995) e Santos (1996).

\section{(ii) Modelos Testados}

Os modelos estatisticos foram selecionados a partir do trabalho de Santos (1996), que testou 34 diferentes modelos em diferentes combinações. 
Tabela 2. Banco de Dados de Biomassa, do INPA $(n=315)$.

(a) Distribuição de Freqüência dos Dados Observados $(n=315)$.

\begin{tabular}{lcl}
\hline Limites de classe & Freq. & $\%$ \\
\hline $5<10$ & 154 & 48,89 \\
$10<20$ & 90 & 28,57 \\
$20<30$ & 28 & 8,89 \\
$30<40$ & 18 & 5,71 \\
$40<50$ & 9 & 2,86 \\
$50<60$ & 8 & 2,54 \\
$60<70$ & 3 & 0,95 \\
$70<80$ & 3 & 0,95 \\
$80<90$ & 0 & - \\
$90<100$ & 1 & 0,32 \\
$100<110$ & 0 & - \\
$110<120$ & 0 & - \\
$120<130$ & 1 & 0,32 \\
\hline total & 315 & 100 \\
\hline
\end{tabular}

(b) Estatística Descritiva dos Dados Observados:

\begin{tabular}{llllll}
\hline variável & média & desvio & CV $(\%)$ & minimo & máximo \\
\hline DAP $(\mathrm{cm})$ & 16,0 & 15,3 & 96 & 5,0 & 120,0 \\
H-total $(\mathrm{m})$ & 17,0 & 7,7 & 45 & 5,6 & 41,4 \\
H-com $(\mathrm{m})$ & 10,7 & 5,2 & 49 & 2,4 & 26,1 \\
P-tronco $(\mathrm{kg})$ & 476,3 & 1299,3 & 273 & 4,5 & 12736,5 \\
P-copa $(\mathrm{kg})$ & 306,4 & 1031,5 & 337 & 0,6 & 12897,9 \\
P-total $(\mathrm{kg})$ & 782,7 & 2271,1 & 290 & 9,1 & 25634,4 \\
copa $(\%)$ & 31 & 14 & 45 & 2 & 70 \\
\hline
\end{tabular}

(c) Estatística Descritiva dos Dados Observados, Divididos em Algumas Classes de Diâmetro:

\begin{tabular}{lccccccc}
\hline $\begin{array}{l}\text { Classes de } \\
\text { diâmetro }\end{array}$ & $\begin{array}{c}\text { número } \\
\text { casos }\end{array}$ & $\begin{array}{c}\text { DAP } \\
\text { média }\end{array}$ & $\begin{array}{r}\text { H- Total } \\
\text { CV }(\%)\end{array}$ & $\begin{array}{c}\text { P - Total } \\
\text { média }\end{array}$ & CV $(\%)$ & média & CV $(\%)$ \\
\hline $5<10$ & 154 & 7,0 & 20 & 11,4 & 27 & 35,7 & 68 \\
$10<15$ & 62 & 12,0 & 12 & 16,4 & 20 & 135,0 & 42 \\
$15<20$ & 28 & 17,5 & 9 & 20,8 & 18 & 407,5 & 34 \\
$20<30$ & 28 & 23,6 & 11 & 23,7 & 13 & 852,0 & 43 \\
$30<50$ & 27 & 37,2 & 14 & 29,3 & 11 & 2449,2 & 35 \\
$>=50$ & 16 & 65,9 & 29 & 34,1 & 10 & 8205,4 & 72 \\
\hline
\end{tabular}


Tabela 3. Dados Utilizados para estudos de Nutrientes $(n=38)$.

(a) Estatística Descritiva dos Dados Observados:

\begin{tabular}{llllll}
\hline variável & média & desvio & CV $(\%)$ & mínimo & máximo \\
\hline DAP $(\mathrm{cm})$ & 39,9 & 20,3 & 51 & 9,5 & 98,0 \\
alt. total $(\mathrm{m})$ & 28,8 & 6,0 & 56 & 11,4 & 41,4 \\
alt. com $(\mathrm{m})$ & 17,3 & 3,7 & 22 & 7,5 & 25,0 \\
P-tronco $(\mathrm{kg})$ & 2147,4 & 2449,1 & 114 & 48,7 & 12736,5 \\
P-copa $(\mathrm{kg})$ & 1595,3 & 2429,5 & 152 & 15,2 & 12898,3 \\
P-total $(\mathrm{kg})$ & 3742,6 & 3005,4 & 128 & 63,9 & 25634,4 \\
copa $(\%)$ & 34 & 14 & 22 & 9 & 63 \\
\hline
\end{tabular}

(b) Contribuição de cada compartimento (tronco, galho grosso, galho fino, folhas e flor/frutos) no peso total de uma árvore $\mathrm{e} \%$ do PF de cada um que è transformado em PS:

\begin{tabular}{llllllll}
\hline PESOS & & tronco & g.grosso & g.fino & folhas & flor/frutos & TOTAL \\
\hline \multirow{3}{*}{ VERDE } & m & 2147,36 & 1109,68 & 434,24 & 50,30 & 1,07 & 3742,61 \\
& s & 2449,14 & 1985,66 & 432,65 & 48,87 & 5,41 & 4793,77 \\
& n & 38 & $38(34)$ & 38 & 38 & $38(8)$ & 38 \\
$\%$ total & m & 65,60 & 17,83 & 14,52 & 2,03 & 0,01 & \\
& s & 14,19 & 13,43 & 7,21 & 1,28 & 0,03 & \\
& n & 38 & $38(34)$ & 38 & 38 & $38(8)$ & \\
SECO & m & 1301,65 & 665,63 & 246,64 & 23,58 & 0,80 & 2238,30 \\
& s & 1552,45 & 1243,55 & 253,6 & 23,01 & 4,60 & 3005,38 \\
& n PF & 38 & $38(34)$ & 38 & 38 & $38(8)$ & 38 \\
& m & 61,11 & 60,56 & 57,22 & 47,56 & 36,73 & 60,28 \\
& s & 8,27 & 7,98 & 5,75 & 7,21 & 20,62 & 7,41 \\
& n & 38 & 34 & 38 & 38 & 8 & 38
\end{tabular}

$\mathrm{m}=$ média aritmética; $\mathrm{s}=$ desvio padrāo amostral; $\mathrm{n}=$ número de observaçōes.

$\%$ total = contribuiçăo do peso de cada compartimento da árvore em relação ao seu peso total. $\% \mathrm{PF}=$ é \% do Peso Fresco da árvore ou do compartimento que corresponde ao Peso Seco.

Tabela 4. Informaçð̃es sobre Densidade da Madeira.

\begin{tabular}{lccccccc}
\hline Espécie & $0 \%$ & $25 \%$ & $50 \%$ & $75 \%$ & $100 \%$ & média & DAP \\
\hline 1 & 0,856 & 0,790 & 0,757 & 0,753 & 0,718 & 0,775 & 0,824 \\
2 & 0,696 & 0,697 & 0,683 & 0,650 & 0,684 & 0,682 & 0,706 \\
3 & 0,879 & 0,903 & 0,866 & 0,741 & 0,724 & 0,823 & 0,913 \\
4 & 0,536 & 0,521 & 0,509 & 0,499 & 0,471 & 0,507 & 0,546 \\
5 & 0,681 & 0,678 & 0,640 & 0,640 & 0,615 & 0,651 & 0,700 \\
6 & 0,818 & 0,807 & 0,806 & 0,653 & 0,704 & 0,758 & 0,838 \\
7 & 0,725 & 0,707 & 0,711 & 0,693 & 0,704 & 0,708 & 0,717 \\
8 & 1,027 & 0,990 & 0,946 & 0,929 & 0,961 & 0,971 & 1,015 \\
9 & 0,891 & 0,870 & 0,862 & 0,862 & 0,846 & 0,866 & 0,896 \\
10 & 0,571 & 0,533 & 0,485 & 0,445 & 0,367 & 0,480 & 0,528 \\
11 & 1,077 & 1,033 & 1,000 & 0,987 & 1,056 & 1,031 & 1,059 \\
12 & 0,891 & 0,870 & 0,807 & 0,716 & 0,846 & 0,826 & 0,896 \\
média & 0,804 & 0,783 & 0,756 & 0,714 & 0,725 & 0,756 & 0,803 \\
desvio & 0,167 & 0,163 & 0,159 & 0,159 & 0,191 & 0,165 & 0,168 \\
mín. & 0,536 & 0,521 & 0,485 & 0,445 & 0,367 & 0,480 & 0,528 \\
máx. & 1,077 & 1,033 & 1,000 & 0,987 & 1,056 & 1,031 & 1,059 \\
\hline
\end{tabular}


$\mathrm{O}$ banco de dados foi dividido em dois, para árvores com $5 \leq \mathrm{DAP}<20 \mathrm{~cm}$ e $\mathrm{DAP} \geq 20 \mathrm{~cm}$. Foram testados os seguintes modelos estatísticos, para todas as árvores com $\mathrm{DAP} \geq 5 \mathrm{~cm}$, equação única, e para as duas classes de tamanho, (a) $5 \leq \mathrm{DAP}<20$ cm e (b) $\mathrm{DAP} \geq 20 \mathrm{~cm}$ :

1. $\ln \mathrm{P}_{\mathrm{i}}=\beta_{0}+\beta_{1} \ln \mathrm{D}_{\mathrm{i}}+\ln \varepsilon_{\mathrm{i}}$ 2. $\ln P_{i}=\beta_{0}+\beta_{1} \ln D_{i}+\beta_{2} \ln H_{i}+\ln \varepsilon_{i}$ 3. $P_{i}=\beta_{0}+\beta_{1} D_{i}^{2} H_{i}+\varepsilon_{i}$ 4. $P_{i}=\beta_{0} D^{\beta 1} H^{\beta 2}+\varepsilon_{i}$ para $\mathrm{i}=1,2, \ldots 315$ - equação única

$$
\begin{aligned}
& i=1,2, \ldots 244 \text { - equação (a) } \\
& i=1,2, \ldots 71 \text { - equação (b) } \\
& \text { onde: }
\end{aligned}
$$

$\mathrm{P}_{\mathrm{i}}=$ peso da massa fresca de cada árvore, em quilograma (para modelos 1,2 e 4) e em toneladas métricas (para o modelo 3 ).

$\mathrm{D}_{\mathrm{i}}=$ diâmetro à altura do peito de cada árvore, DAP, em centímetros (para modelos 1,2 e 4) e em metros (para o modelo 3) em metros

$\mathrm{H}_{\mathrm{i}}=$ altura total de cada árvore, regressão

$\beta_{0}, \beta_{1}$ e $\beta_{2}=$ coeficientes de

$$
\begin{aligned}
& \varepsilon_{\mathrm{i}}=\text { erro aleatório } \\
& \ln =\text { logaritmo natural }
\end{aligned}
$$

Os modelos estatísticos propostos por Brown e Lugo (Foster Brown et al., 1995; Salomão et al., 1996) e aqueles que apresentaram os melhores resultados no trabalho de Saldarriaga et al. (1988), que incluem densidade da madeira, não foram testados porque esta variável é de dificil obtenção para cada indivíduo em pé. Além disso, segundo Higuchi \& Carvalho Jr. (1994), a densidade da madeira $\left(\mathrm{g} / \mathrm{cm}^{3}\right)$ apresenta significativas variações intra e inter-especificas. Pelas mesmas razões, Overman et al. (1994) descartam esta variável, apesar do bom desempenho dos modelos que a contém.

$\mathrm{Na}$ tabela 4 , onde visualizam-se as densidades de 12 árvores, observa-se que: a menor densidade é de 0,480 e a maior é de 1,031; a densidade tende a diminuir no sentido base-topo; a densidade média, considerando base-topo, é de 0,756; e esta última variável é sempre menor que a densidade média obtida na altura do DAP. A densidade média do DAP é igual a 0,803 , que, por sua vez, é diferente de todas as estimativas fornecidas por Foster Brown et al. (1995) e a de Saldarriaga et al. (1988). As variações no sentido casca-medula também são significativas (Higuchi \& Carvalho Jr., 1994).

\section{(iii) Escolha do Melhor Modelo Estatístico:}

Para a escolha do melhor modelo estatístico visando-se estimar a biomassa em pé da área em estudo, foram adotados os procedimentos tradicionais da ciência florestal, que são: maior coeficiente de determinação, menor erro padrão de estimativa e melhor distribuição dos resíduos (Santos, 1996). Além destes procedimentos, foram simuladas amostras de diferentes intensidades, para testar a consistência dos modelos na estimativa da biomassa. Foram tomadas 15 amostras com 50 árvores selecionadas aleatoriamente do banco de dados original; 10 amostras com $n=100 ; 5$ amostras com $\mathrm{n}=200$; e 5 amostras com $\mathrm{n}=300$.

\section{Resultados e Discussão}

Do trabalho de Higuchi \& Carvalho Jr. (1994), as seguintes informações quantitativas do sitio estudado são importantes para uma melhor interpretação 
destes resultados e para futuras comparações com outros sítios:

- Em uma parcela fixa de $2.000 \mathrm{~m}^{2}$, o peso da biomassa fresca distribui-se da seguinte maneira, em relação ao peso total: a vegetação (exceto cipós) com DAP $\geq 5 \mathrm{~cm}$ contribui com $86,9 \%$ do peso total; a vegetação com DAP $\angle 5 \mathrm{~cm}$ contribui com $2,4 \%$; os cipós contribuem com $1,3 \%$ e a liteira (toda a vegetação morta sobre a superficie do solo) contribui com $9,4 \%$.

- Os teores médios de carbono são os seguintes: tronco (48\%), galhos grossos $(48 \%)$, galhos finos $(47 \%)$, folhas (39\%), plântulas - até $50 \mathrm{~cm}$ de altura - $(47 \%)$, mudas - altura $>50 \mathrm{~cm}$ e DAP $<5 \mathrm{~cm}$ $(49 \%)$, cipós $(48 \%)$ e liteira $(39 \%)$.

Os coeficientes de regressão e de determinação e os erros padrões de estimativa de todos os quatro modelos estatísticos testados (árvores com DAP $\geq 5 \mathrm{~cm}$ ), incluindo as variações (a) para árvores com $5 \leq \mathrm{DAP}<20 \mathrm{~cm}$ e $(b)$ $\mathrm{DAP} \geq 20 \mathrm{~cm}$, verificam-se na tabela 5 . De um modo geral, os quatro modelos (incluindo as variações $a$ e $b$ ) estão aprovados nos quesitos coeficiente de determinação $\left(\mathrm{r}^{2}\right)$ e erro padrão de estimativa $\left(\mathrm{s}_{\mathrm{y} . \mathrm{x}}\right) \mathrm{e}$, por esta razão, poderiam ser utilizados para estimar a biomassa de árvores em pé da área em estudo.

Todos os modelos apresentam coeficientes de correlação (r) altamente significantes $(\alpha<0,01)$. De um modo geral, os modelos únicos para árvores com DAP $\geq 5 \mathrm{~cm}$ apresentam os maiores coeficientes de determinação $\left(r^{2}\right)$, exceto para o modelo 3. Com relação ao $\left(\mathrm{s}_{\mathrm{yx}}\right), \mathrm{o}$ modelo 4 é o que tem o melhor desempenho, apresentando os menores erros, seguido do modelo 2 . Combinando as equações $\boldsymbol{a}$ e $\boldsymbol{b}$, no mesmo banco de
Tabela 5. Coeficientes de Regressão e de Determinação, Erro Padrão de Estimativa dos Modelos Estatísticos para Estimar a Biomassa (Peso total) de Árvores em pé.

\begin{tabular}{lccccc}
\hline Modelo & $\mathrm{b}_{0}$ & $\mathrm{~b}_{1}$ & $\mathrm{~b}_{2}$ & $\mathrm{r}^{2}$ & $\mathrm{~s}_{\gamma, \mathrm{x}}$ \\
\hline 1 & $-1,497$ & 20,548 & & 0,97 & 1729 \\
$1 \mathrm{a}$ & $-1,754$ & 2,665 & & 0,92 & 43 \\
$1 \mathrm{~b}$ & $-0,151$ & 2,170 & & 0,90 & 2035 \\
2 & $-2,694$ & 2,038 & 0,902 & 0,98 & 812 \\
$2 \mathrm{a}$ & $-2,668$ & 2,081 & 0,852 & 0,95 & 35 \\
$2 \mathrm{~b}$ & $-2,088$ & 1,837 & 0,939 & 0,91 & 1497 \\
3 & 0,077 & 0,492 & & 0,90 & 716 \\
$3 \mathrm{a}$ & 0,0056 & 0,621 & & 0,94 & 34 \\
$3 \mathrm{~b}$ & 0,393 & 0,473 & & 0,86 & 1508 \\
4 & 0,001 & 1,579 & 2,621 & 0,94 & 540 \\
$4 \mathrm{a}$ & 0,0336 & 2,171 & 1,038 & 0,94 & 31 \\
$4 \mathrm{~b}$ & 0,0009 & 1,585 & 2,651 & 0,92 & 1159 \\
\hline
\end{tabular}

$b_{0}, b_{1}$ e $b_{2}=$ estimadores dos parâmetros $\beta_{0}, \beta_{1}$ e $\beta_{2}$, respectivamente.

$r^{2}=$ coeficiente de determinação ajustado

$r_{y x}=$ erro padrăo de estimativa.

dados, os erros (em quilogramas) produzidos foram: $949,693,356$ e 537 , respectivamente para os modelos $1,2,3 \mathrm{e}$ 4. Nesta situação, o melhor desempenho é do modelo 3 , seguido do modelo 4 .

$\mathrm{O}$ exame da distribuição dos resíduos mostra que os modelos 1,2 e 3 não apresentam nenhum padrão, distribuindo-se aleatoriamente ao longo do eixo da biomassa observada e estimada, ordenada de forma crescente pela variável DAP. O modelo 4, no entanto, apresenta um claro padrão, aumentando os desvios conforme aumentam os DAP's.

\section{As equações resultantes são:}

Modelo 1:

- Equações a \& b:

(a) $\ln P=-1,754+2,665 \ln D$; para $5 \leq \mathrm{DAP}<20 \mathrm{~cm}$

(b) $\ln P=-0,151+2,170 \ln D$; para DAP $\geq 20 \mathrm{~cm}$

- Equação única:

$\ln P=-1,497+2,548 \ln D$; para $\mathrm{DAP} \geq 5 \mathrm{~cm}$

Modelo2:

- Equações a \& b: 
(a) $\ln P=-2,668+2,081 \ln D+0,852$ In $H$; para $5 \leq \mathrm{DAP}<20$

(b) $\ln P=-2,088+1,837 \ln D+0,939$ In $H$; para $\mathrm{DAP} \geq 20 \mathrm{~cm}$

- Equação única: $\ln P=-2,694+2,038$ $\ln D+0,902 \ln H$; para DAP $\geq 5 \mathrm{~cm}$ Modelo 3:

- Equações a \& b:

(a) $P=0,0056+0,621 D^{2} H$; para $0,05 \leq \mathrm{DAP}<0,20 \mathrm{~m}$

(b) $P=0,393+0,473 D^{2} H$; para DAP $\geq 0,20 \mathrm{~m}$

- Equação única: $P=0,077+0,492$

$D^{2} H$; para DAP $\geq 0,05 \mathrm{~m}$

Modelo 4:

- Equações a \& b:

(a) $P=0,0336 * D^{2,171} * H^{1,038}$; para $5 \leq$ DAP $<20 \mathrm{~cm}$

(b) $P=0,0009 * D^{1,585 *} H^{2,651}$; para $\mathrm{DAP} \geq 20 \mathrm{~cm}$

- Equação única: $P=0,001$ * $D^{l, 579 *} H^{2,62 l}$; para DAP $\geq 5 \mathrm{~cm}$

A verificação da consistência de cada um dos modelos estatísticos para estimar a biomassa em pé, sobre amostras simuladas (tiradas aleatoriamente do banco de dados original), encontram-se na tabela 6 . Nesta tabela verificam-se as médias observadas e estimadas em cada simulação. A análise é feita sobre o afastamento da média estimada em relação à observada, em percentagem, utilizando-se equações distintas para estimar a biomassa de árvores com $5 \leq$ DAP $<20 \mathrm{~cm}$ e DAP $\geq 20 \mathrm{~cm}$ e uma única equação para todas as árvores contidas na amostra com DAP $\geq 5 \mathrm{~cm}$.

\section{(i) Modelo 1}

- Usando as equações $a$ e $b$, para estimar a biomassa do banco de dados original, a média estimada afasta-se $1,9 \%$ da média observada, ou seja, o desvio ${ }^{3}$ é de $-1,9 \%$. Quando utiliza-se uma só equação para estimar a biomassa das duas classes de diâmetro, o desempenho anterior não é repetido, apresentando um desvio de $+16 \%$. Excepcionalmente, na simulação com $n=50$, o uso de uma só equação resulta em um desvio médio de $+2,8 \%$, que poderia ser considerado bom se não fosse a amplitude de variação entre o menor $\mathrm{e} o$ maior desvio, que foi de 0,1 a $24,9 \%$.

- Este modelo (equações $a$ e $b$ ) demonstra a mesma consistência nas simulações $\operatorname{com} n=300, n=200$ e $n=$ 100 , respectivamente, com desvios de $1,9 \%$ (1,6 e 2,3, menor e maior desvio, em valores absolutos), $+0,5 \%(2,7$ e 11,6$)$ e $+2,6 \%(3,7$ e 22,1). A simulação com $n=$ 50 , o desvio médio é de $-10,2 \%$.

- A equação única para estimar a biomassa, usando este modelo estatístico, não é alternativa para as duas equações, ou seja, o uso deste modelo requer as duas equações para estimar a biomassa de árvores com $5 \leq \mathrm{DAP}<20 \mathrm{~cm}$ e DAP $\geq$ $20 \mathrm{~cm}$, separadamente.

- Trata-se de um modelo com apenas o DAP como variável independente, que é uma variável fácil de ser medida no campo, sem erros não amostrais. $\mathrm{O}$ único problema deste modelo é que o peso será sempre o

3 Desvio é afastamento, em \%, do peso médio estimado pelas diferentes equações, em relação ao peso médio observado. Entre parêntesis, os desvios aparecem em valores absolutos e o primeiro é sempre o menor e, o segundo, o maior desvio. 
Tabela 6. Resumo das simulações utilizando diferentes intensidades de amostragem (tomadas aleatoriamente do banco de dados).

\begin{tabular}{|c|c|c|c|}
\hline & \multirow{2}{*}{$\frac{\text { Biomassa }}{\text { (observada e estimada) }}$} & \multicolumn{2}{|c|}{ observada } \\
\hline & & equações $a \& b$ & equação única \\
\hline $\begin{array}{l}\text { banco de dados } \\
(n=315)\end{array}$ & $\begin{array}{l}\text { observada } \\
\text { modelo } 1 \\
\text { modelo } 2 \\
\text { modelo } 3 \\
\text { modelo } 4 \\
\end{array}$ & $\begin{array}{l}768,2[-1,9] \\
754,6[-3,6] \\
792,1[+1,2] \\
746,9[-4,6]\end{array}$ & $\begin{array}{l}907,7[+16,0] \\
805,2[+2,9] \\
783,3[+0,1] \\
725,3[-7,3]\end{array}$ \\
\hline $\begin{array}{l}\text { amostra com } n=300 \\
\text { (5 repetiçôes) }\end{array}$ & $\begin{array}{l}\text { observada } \\
\text { modelo } 1 \\
\text { modelo } 2 \\
\text { modelo } 3 \\
\text { modelo } 4 \\
\end{array}$ & $\begin{array}{l}779,1[-1,9] \\
765,5[-3,6] \\
803,3[+1,2] \\
760,2[-4,3]\end{array}$ & $\begin{array}{l}4,1 \\
924,1[+16,4] \\
817,0[+2,9] \\
794,7[+0,1] \\
738,9[-7,0] \\
\end{array}$ \\
\hline $\begin{array}{l}\text { amostra com } n=200 \\
\text { (5 repetiçőes) }\end{array}$ & $\begin{array}{l}\text { observada } \\
\text { modelo } 1 \\
\text { modelo } 2 \\
\text { modelo } 3 \\
\text { modelo } 4\end{array}$ & $\begin{array}{l}788,3[+0,5] \\
770,0[-1,8] \\
808,1[+3,1] \\
786,3[+0,3]\end{array}$ & $\begin{array}{l}4,2 \\
944,2[+20,4] \\
826,4[+5,4] \\
801,3[+2,2] \\
740,2[-5,6]\end{array}$ \\
\hline $\begin{array}{l}\text { amostra com } n=100 \\
\text { (10 repetiçőes) }\end{array}$ & $\begin{array}{l}\text { observada } \\
\text { modelo } 1 \\
\text { modelo } 2 \\
\text { modelo } 3 \\
\text { modelo } 4\end{array}$ & $\begin{array}{l}866,9[+2,6] \\
835,4[-1,1] \\
876,6[+3,8] \\
811,3[-4,0]\end{array}$ & $\begin{array}{l}4,8 \\
1052,4[+24,6] \\
900,5[+6,6] \\
865,1[+2,4] \\
790,8[-6,4]\end{array}$ \\
\hline $\begin{array}{l}\text { amostra com } n=50 \\
\text { (15 repetições) }\end{array}$ & $\begin{array}{l}\text { observada } \\
\text { modelo } 1 \\
\text { modelo } 2 \\
\text { modelo } 3 \\
\text { modelo } 4 \\
\end{array}$ & $\begin{array}{l}750,8[-10,2] \\
757,2[-9,4] \\
795,8[-4,8] \\
771,8[-7,7]\end{array}$ & $\begin{array}{l}6,2 \\
859,3[+2,8] \\
799,8[-4,4] \\
779,1[-6,8] \\
750,8[-10,2] \\
\end{array}$ \\
\hline
\end{tabular}

mesmo, para um determinado diâmetro, independentemente da altura da árvore, da espécie e de outros atributos da árvore.

\section{(ii) Modelo 2}

- Usando as equações $\boldsymbol{a}$ e $\boldsymbol{b}$, para estimar a biomassa do banco de dados original, a média estimada afasta-se $3,6 \%$ da média observada. Quando utiliza-se uma só equação para estimar a biomassa das duas classes de diâmetro, o seu desempenho é melhor do que o anterior, com desvio de $+2,9 \%$.

- Este modelo (equações $a$ e $b$ ) demonstra a mesma consistência nas simulações $\operatorname{com} n=300, n=200$ e $n$
$=100$, respectivamente, com desvios de $-3,6 \%$ (3,2 e 4,3, menor e maior desvio, em valores absolutos), $-1,8 \%$ $(5,2$ e 6,7$)$ e $-1,1 \%(0,9$ e 12,7$)$. A simulação com $\mathrm{n}=50$, o desvio médio é de $-9,4 \%$. O uso de uma só equação tem um desempenho razoável para todas as simulações, exceto para $\mathrm{n}=$ 50 , que apresenta desvio menor do que quando utiliza-se as equações $a$ e $b$.

- Apesar do bom desempenho da equação única, em relação aos desvios médios, onde as diferenças são negligiveis, as amplitudes de variação dos mesmos nas equações $\boldsymbol{a}$ e $\boldsymbol{b}$ são menores, sendo, por esta razão, mais apropriadas para a estimativa da biomassa. 
- A incorporação da altura total neste modelo permite estimar diferentes pesos para iguais DAP's, ao contrário do modelo 1.

\section{(iii) Modelo 3}

- Usando as equações $a$ e $b$, para estimar a biomassa do banco de dados original, a média estimada afasta-se $+1,2 \%$ da média observada. Quando se utiliza uma só equação para estimar a biomassa das duas classes de diâmetro, o seu desempenho é melhor do que o anterior, com desvio de $+0,1 \%$. Apesar de um claro padrão na distribuição dos resíduos, este modelo tem uma boa capacidade de compensação quando se utiliza todo o banco de dados, tanto com as equações $a$ e $b$ como com a equação única para as duas classes de diâmetro.

- Este modelo (equações $a$ e $b$ ) demonstra a mesma consistência nas simulações $\operatorname{com} n=300, n=200, n=100$ e $\mathrm{n}=50$, respectivamente, com desvios de $+1,2 \%(0,4$ e 1,6 , menor e maior desvio, em valores absolutos), $+3,1 \%(1,1$ e 13,7), $+3,8 \%(0,8$ e 20,3$)$ e $-4,8 \%(0,4$ e 19,4$)$. $\mathrm{O}$ uso de uma só equação tem um desempenho tão consistente quanto ao anterior, com desvios de $+0,1 \%(0,2$ e 0,9$)$, $+2,2 \%(0,6$ e 11,5$),+2,4 \%(0,7$ e 17,6$) \mathrm{e}-$ $6,8 \%(0,4$ e 16,2$)$, respectivamente para $n$ $=300, n=200, n=100$ e $n=50$.

- A equação única para este modelo é a melhor alternativa para estimar a biomassa, principalmente considerando apenas a estimativa da biomassa média de uma parcela fixa, sem preocupar-se com as estimativas individuais. Em todos os tamanhos da amostragem, esta equação demonstrouse bastante consistente e precisa.
- Sem preocupar-se com as estimativas individuais, prestando atenção apenas no total ou na média das parcelas fixas, este é o melhor modelo entre os testados. De um modo geral, este modelo superestima o peso das menores classes de diâmetro. Para grandes inventários para estimativa de biomassa, este modelo é o mais preciso.

\section{(iv) Modelo 4}

- Usando as equações $\boldsymbol{a}$ e $\boldsymbol{b}$, para estimar a biomassa do banco de dados original, a média estimada afasta-se $4,6 \%$ da média observada. Quando utiliza-se uma só equação para estimar a biomassa das duas classes de diâmetro, o desempenho anterior não é repetido, com desvio de $-7,3 \%$.

- Este modelo (equações $a$ e $b$ ) demonstra a mesma consistência nas simulações com $n=300, n=200, n=100$ e $n=50$, respectivamente, com desvios de $-4,3 \%$ (3,4 e 5,1, menor e maior desvio, em valores absolutos), $+0,3 \%(0,6$ e 3,7$)$, $-4,0 \%(1,2$ e 7,6$)$ e $-7,7 \%(4,2$ e 16,1$)$. O uso de uma só equação tem um desempenho inferior a todos os outros modelos testados e, por esta razão, não é uma alternativa para as duas equações. Neste caso, a opção tem que ser pelas duas equações, $4 a$ para árvores com $5 \leq \mathrm{DAP}$ $<20 \mathrm{~cm} \mathrm{e} 4 b$ para DAP $\geq 20 \mathrm{~cm}$.

- De todos os modelos testados, este modelo é o que apresenta as menores amplitudes de variação, demonstrando uma boa consistência na estimativa da biomassa. É um modelo bastante conservador e que apresenta poucas surpresas na estimativa da biomassa das diferentes classes de diâmetro. 


\section{Considerações finais}

1. Os quatro modelos estatísticos testados produzem estimativas confiáveis de biomassa de árvore em pé, todos com desvios inferiores a $5 \%$ em relação à média.

2. As equações distintas para árvores com $5 \leq \mathrm{DAP}<20 \mathrm{~cm}$ e com DAP $\geq 20 \mathrm{~cm}$ são mais consistentes que a equação única para todas as árvores com DAP $\geq 5 \mathrm{~cm}$.

3. Dentre os modelos testados, os melhores são os modelos 1 e 4 , respectivamente com as seguintes equações:

(a) $\ln P=-1,754+2,665 \ln D$; para $5 \leq \mathrm{DAP}<20 \mathrm{~cm}$

(b) $\ln P=-0,151+2,170 \ln D$; para DAP $\geq 20 \mathrm{~cm}$

\section{e}

(a) $P=0,0336 * D^{2,171 *} H^{1,038}$; para 5 DAP $<20 \mathrm{~cm}$

(b) $P=0,0009 * D^{1,585 *} H^{2,651}$; para DAP $\geq 20 \mathrm{~cm}$

- O modelo 1 tem a vantagem de ser dependente de apenas uma variável, o DAP, que é uma variável fácil de ser medida no campo, com pouco risco de erros não amostrais;

- O modelo 4 tem a vantagem de ser muito consistente e de poder estimar mais realisticamente árvores individuais, com mesmos DAP's e diferentes alturas. Além disso, este modelo já foi preliminarmente utilizado por Araujo (1995), em ToméAçu (Pará), para confrontar com os resultados obtidos pelo método direto. Em Tomé-Açu, a biomassa estimada por este modelo ficou também a menos de $5 \%$ da observada.

4. A eficiência das equações está associada à utilização de parcelas fixas para o inventário de biomassa de um determinado sitio, com as dimensões minimas recomendadas para os inventários florestais na Amazônia.

5. O peso do tronco seco corresponde a $61 \%$ de seu peso antes da secagem; e o da copa corresponde a $58 \%$ de seu peso fresco.

6. Do peso total de uma árvore, $65,6 \%$ é tronco e $34,4 \%$ é copa. A contribuição de cada compartimento da árvore em seu peso total é a seguinte: tronco $(65,6 \%)$, galho grosso $(17,8 \%)$, galho fino $(14,5 \%)$, folhas $(2,03 \%)$ e flores/frutos $(0,01 \%)$.

7. Os teores médios de carbono são os seguintes: tronco (48\%), galhos grossos (48\%), galhos finos (47\%) e folhas $(39 \%)$.

- modelo 1: $\ln \mathrm{P}_{\mathrm{i}}=\mathrm{b}_{0}+\mathrm{b}_{1} \ln \mathrm{D}_{\mathrm{i}}$; sendo (1) para DAP $\geq 5 \mathrm{~cm} \mathrm{e} \mathrm{i}=1, \ldots, 315$; (1a) para $5 \leq \mathrm{DAP}<20 \mathrm{~cm}$ e $\mathrm{i}=1, \ldots, 244$; e (1b) para DAP $\geq 20 \mathrm{~cm}$ e $\mathrm{i}=1, \ldots, 71$. - modelo 2; $\ln \mathrm{P}_{\mathrm{i}}=\mathrm{b}_{0}+\mathrm{b}_{1} \ln \mathrm{D}_{\mathrm{i}}+\mathrm{b}_{2} \ln \mathrm{H}_{\mathrm{i}}$; sendo (2) para DAP $\geq 5 \mathrm{~cm} \mathrm{e} \mathrm{i}=1, \ldots ., 315$; (2a) para $5 \leq \mathrm{DAP}<20 \mathrm{~cm} \mathrm{ei}=1, \ldots, 244$; e (2b) para $\mathrm{DAP} \geq 20 \mathrm{~cm}$ e $\mathrm{i}=1, \ldots, 71$. - modelo 3: $\mathrm{P}_{\mathrm{i}}=\mathrm{b}_{0}+\mathrm{b}_{1} \mathrm{D}_{\mathrm{i}}^{2} \mathrm{H}_{i}$; sendo (3) para DAP $\geq 0,05 \mathrm{~m}$ e $\mathrm{i}=1, \ldots, 315$; (3a) para $0,05 \leq \mathrm{DAP}<0,20 \mathrm{me} \mathrm{i}=1, \ldots, 244$; e (3b) para DAP $\geq 0,20 \mathrm{~m} \mathrm{e} \mathrm{i}=1, \ldots, 71$. - modelo 4: $\mathrm{P}_{\mathrm{i}}=\mathrm{b}_{0} \mathrm{D}^{\mathrm{bl}} \mathrm{H}^{\mathrm{b} 2}$; sendo (1) para $\mathrm{DAP} \leq 5 \mathrm{~cm}$ e $\mathrm{i}=1, \ldots, 315$; (1a) para $5 \leq \mathrm{DAP}<20 \mathrm{~cm} \mathrm{e} \mathrm{i}=1, \ldots, 244$; e (lb) para $\mathrm{DAP} \geq 20 \mathrm{~cm}$ e $\mathrm{i}=1, \ldots, 71$.

\section{Bibliografia citada}

Araújo, T.M. 1995. Investigação das Taxas de Dióxido de Carbono Gerado em Queimadas na Regiâo Amazônica. Tese de Doutorado, Universidade Estadual 
Paulista, Faculdade de Engenharia de Guaratinguetá, 212 p..

Carvalho Jr., J.A; J.M. Santos, J.C. Santos M.M. Leitão; N. Higuchi. 1995. A Tropical Rainforest Clearing Experiment by Biomass Buming in the Manaus Region. Atmospheric Environment, 29(17):2301-2309.

Fearnside, P.M.; N. Leal Filho ; F.M Fernandes. 1993. Rainforest Burning and the Global Budget: Biomass, Combustion Efficiency, and Charcoal Formation in the Brazilian Amazon. J. of Geophysical Research, 98(D9):16733-16743.

Fearnside, P.M. 1996. Amazonian Deforestation and Global Warming: Carbon Stocks in Vegetation Replacing Brazil's Amazon Forest. Forest Ecology and Management. 80:21-34

Foster Brown, I.; D.C. Nepstad; I.O. Pires, L.M. Luz e A.S. Alechandre. 1992. Carbon Storage and Land-use in Extractive Reserves, Acre, Brazil. Environmental Conservation, 19(4):307-315.

Foster Brown, 1.; Martinelli, L.A.; Wayt Thomas, W.; Moreira, M.Z.; Cid Ferreira, C.A.; Victoria, R.A. 1995. Uncertainty in the Biomass of Amazonian Forests: an Example from Rondônia, Brazil. Forest Ecology and Management, 75:175-189.

Higuchi, N.; Carvalho Jr., J.A. 1994. Fitomassa e Conteúdo de Carbono de Espécies Arbóreas da Amazônia. : Anais do Seminário "Emissão x Seqüestro de $\mathrm{CO}$, - Uma Nova Oportunidade de Negócios para o Brasil":127-153.
Higuchi, N.; Santos, J.M.; Imanaga, M.; Yoshida, S. 1994. Aboveground Biomass Estimate for Amazonian Dense Tropical Moist Forests. Memoirs of the Faculty of Agriculture, Kagoshima, 30(39):43-54.

Overman, J.P.M.; Witte,H.J.L.; Saldarriaga, J.G.. 1994. Evaluation of Regression Models for Above-ground Biomass Determination in Amazonia Rainforest. Forest Ecology and Management, 10:207-218.

Saldarriaga, J.G.; West, D.C.; Tharp, M.L.; Uhl, C. 1988. Long-term Chronosequence of Forest Sucession in the Upper Rio Negro of Colombia and Venezuela. Journal of Ecology 76:938-958.

Salomão, R.P; Nepstad, D.C.; Vieira, I.C.G.. 1996. Como a Biomassa de Florestas Tropicais Influi no Efeito Estufa. Ciencia Hoje, 21(122):38-47.

Santos, J. dos. 1996. Análise de Modelos de Regressão para Estimar a Fitomassa da Floresta Tropical Úmida de Terra-firme da Amazônia Brasileira. Tese de Doutorado, Universidade Federal de Viçosa, 121 p..

Schroeder, P.E.; Winjum, J.K.. 1995. Assessing Brazil's Carbon Budget: II. Biotic Fluxes and Net Carbon Balance. Forest Ecology and Management, 75(87-99).

Skole, D.L.; Chomentowski, W.H..; Salas, W.A.; Nobre, A.D. 1994. Physical and Human Dimensions of Deforestation in Amazonia. BioScience, 44(5):314-321. 